\title{
Frontières
}

\section{L’antidépresseur et le mal de vivre}

\section{Catherine Mavrikakis et Christian Saint-Germain}

Volume 21, numéro 2, printemps 2009

Détresse psychique et antidépresseurs

URI : https://id.erudit.org/iderudit/039450ar

DOI : https://doi.org/10.7202/039450ar

Aller au sommaire du numéro

Éditeur(s)

Université du Québec à Montréal

ISSN

1180-3479 (imprimé)

1916-0976 (numérique)

Découvrir la revue

Citer ce document

Mavrikakis, C. \& Saint-Germain, C. (2009). L’antidépresseur et le mal de vivre. Frontières, 21(2), 7-8. https://doi.org/10.7202/039450ar d'utilisation que vous pouvez consulter en ligne.

https://apropos.erudit.org/fr/usagers/politique-dutilisation/ 


\section{L'ANTIDÉPRESSEUR ET LE MAL DE VIVRE}

\author{
Catherine Mavrikakis, \\ directrice du numéro. \\ Christian Saint-Germain, \\ directeur du numéro.
}

On peut dire que dans la modernité - pour paraphraser une formule freudienne - que là où la philosophie était, la pharmacologie est advenue. La poursuite du bonheur, l'injonction à participer à tout le dispositif de la consommation n'a jamais pesé aussi lourd sur la conscience des citoyens. Dans ce contexte, la tristesse, le deuil, la mélancolie ne sont pas de mise, en tout cas, elles ne doivent pas se prolonger. Tout un marché de l'« antidépression» instaure sa mainmise sur l'humeur, cette entité mystérieuse dont les mouvements sont, à l'instar de l'économie et de la météo, hautement fluctuants pour les sujets. Il s'agit de vivre au beau fixe, dût-on accepter le paysage statique d'un soi prévisible, distant ou analgésié.

Ce numéro de la revue Frontières offre une facette, un point de vue sur la complexité des intrications entre le soin pharmacologique, la détresse et l'antidépresseur. Il n'a pas la prétention d'être exhaustif tant les enjeux des molécules dépassent la pharmacologie elle-même. La poursuite du bonheur est d'ailleurs une chose trop sérieuse pour l'abandonner au pharmacien ou même au philosophe. Jamais une époque ne s'est autant préoccupée du bonheur de l'homme ordinaire ni ne l'a autant confondu - derrière la définition ambiguë de santé mentale - avec la normalité. La conception médicale de la «santé psychique » et éventuellement son rétablissement par voie moléculaire sont parfaitement assortis à l'idéal consumériste. Si Freud voulait faire accéder par son écoute le névrosé à un «malheur ordinaire», il n'avait pas envisagé la démocratisation du «bonheur». Or, l'antidépresseur pourvoit peut-être, à l'instar du téléviseur à écran plat, au bonheur des masses repues.

La section Articles de ce numéro s'ouvre sur un texte de Maurice Boutin intitulé: «Fluctuations du mal-être et gestion de l'humeur » qui pose les enjeux théoriques et philosophiques qui nous guideront ici. Cet article installe, si l'on peut dire, l'horizon humoral et la critique de l'être humain "administré» jusque dans les recoins les plus intimes de l'intériorité, en passant par l'antidépresseur et le Viagra. L'auteur montre l'incertitude croissante, l'ambivalence du moi dépendant et l'établissement d'un style de vie. Plus précisément, il arrive à décrire l'effritement du consensus social quant aux pratiques et aux conceptions de ces nouveaux rapports à un soi médicamenté. La modernité fabriquerait désormais un individu sous influence sans trop d'égards pour la réalité diagnostique du trouble.

L'article de Christian Saint-Germain tente de problématiser le paysage éthique inédit issu de l'argumentation de la consommation d'antidépresseurs et décrit les conséquences théoriques pour le sujet éthique de l'élargissement des usages de l'antidépresseur. C'est toute la mécanique de l'imputabilité, l'arrangement de la causalité subjective qui sont bouleversés lorsque le sujet éthique frappe le mur d'une nouvelle réalité moléculaire, celle de l'antidépresseur. C'est que, pour être efficace, ce médicament doit essentiellement désinhiber l'action. Comment alors parler d'imputabilité et de libre arbitre si le dépressif - sans antécédent criminel ou de comportements violents - n'est plus tout à fait lui-même, ne se possède pas complètement? Comment relier les actes d'un déprimé sous médication à son intention véritable? La reconfiguration de la subjectivité moderne oblige l'éthique à reconsidérer l'engrenage de la mise en accusation et de la responsabilité pénale. L'époque de la médicalisation de la conscience s'ouvre sur la scène subjective d'une culpabilité paradoxale: sujets responsables, mais non pas coupables.

Dans «Les visages de l'antidépresseur. Pathologisation du corps féminin », Catherine Mavrikakis montre comment l'imaginaire de l'antidépresseur et de l'overdose travaille le désir pour la playmate, celle qui est perçue comme la plus belle femme, l'objet sexuel. Il semblerait en effet que ce qui ravit le spectateur des beautés telles que Marylin Monroe ou Anna Nicole Smith, c'est leur fragilité psychique, leur état dépressif et la possibilité de les voir mourir d'une prise mal gérée de médicaments censés guérir l'âme. Bien que l'image publicitaire, à l'heure actuelle, valorise un visage détendu de femme qui se soigne à la fois moralement et physiquement, le désir tel qu'il se montre dans la consommation d'images érotiques et pornographiques s'articule autour d'une pathologisation de la femme et du médicament comme puissance érotique.

À travers l'étude de deux films récents, Hard Candy (2005) et The Brave One (2007), qui s'inscrivent dans une relecture du Petit chaperon rouge, Martine Delvaux poursuit la réflexion sur l'antidépresseur et les femmes amorcée dans l'article de Mavrikakis. Les films ici analysés présentent une fille et une femme qui deviennent meurtrières à la suite de violences qu'elles ont subies. Si ces femmes semblent souffrantes mentalement, la détresse psychique qui les caractérise, dans un premier temps, n'est pas ici éliminée, domestiquée, mais "devient le moteur d'une rage vengeresse, d'une immense colère ». C'est sur le droit à cette colère et au meurtre plutôt qu'au médicament régulateur des émotions et anesthésiant que Delvaux réfléchit ici à travers une prise de position sur la représentation des femmes.

Sarah-Anaïs Crevier Goulet s'inscrit elle aussi dans une réflexion sur le féminin et la dépression. Dans l'article, "Malcastrée" et "médiquée". Emma Santos, entre folie et dépression ", l'auteure nous fait connaître une écrivaine violemment passionnante qui, dans les années 1970, a publié huit livres peu lus jusqu'ici. Ces derniers relatent les années de dépression de Santos passées en maison de santé ou dans la solitude. Dans l'écriture, Santos tente de résister à l'emprise des médicaments et à «tenir». À travers l'expérience centrale de la perte, Santos, par ses textes, «s'accroche aux mots » et fait obstacle au dispositif institutionnel qui veut la «soigner» et la réduire au silence. 
Dans « Petit traité du devenir rien à travers quelques figures féminines durassiennes ", Marie-Soleil Roy, en faisant écho aux propos que l'on retrouve sous la plume de Crevier Goulet, se penche sur la question de l'écriture et du désespoir psychique. Réfléchissant sur trois œuvres du corpus de l'écrivaine française Marguerite Duras, soit Le Vice-consul (1966), Détruire dit-elle (1969) et India Song (1973), Roy veut penser les liens «entre la détresse et la mise en récit du corps». Dans un monde de corps maigres, acharnés à disparaître, à devenir rien, le livre apparaît pour Duras comme une suspension de la mort, un philtre à absorber qui peut aussi s'avérer un poison dangereux.

L'article de Martin Tailly «De la mélancolie considérée comme remède» introduit à la dimension métaphysique de la consommation des «soleils factices de la pharmaceutique». Résolument critique à l'égard d'une médicalisation du malaise, il s'agit pour l'auteur de déplacer vers le continent «mélancolique» l'affection dépressive. En reprenant les positions de Thomas Bernhard et de Theodor W. Adorno, il ne peut plus être question de tenter de survivre par expédient moléculaire ou pis-aller ontologique, mais il s'agit d'accepter la mélancolie. En d'autres mots, «constamment induire en soi un état d'esprit qui soit propre au penser lucide et non pas à la paix intérieure». Une pareille ouverture correspond parfaitement depuis Aristote à l'activité philosophique dans la tradition occidentale. Il n'est guère de penseur important qui n'ait éprouvé les effets de la «bile noire » sur son tempérament. L'auteur assume la tonalité mélancolique depuis le va-et-vient théorique qu'il opère entre Søren Kierkegaard et Thomas Bernhard. Il en va ainsi d'une justification de l'état mélancolique non plus compris dans son élément pathologique, mais comme une dimension essentielle du penser et de l'écrire lui-même, de la mélancolie comme «médicament de survie».

S'interrogeant d'emblée sur le pouvoir réparateur de la culture et de la littérature dans une réflexion sur la «nécessité du désespoir » dans la sphère littéraire et artistique, l'article de Frédérique Bernier, «Lire désespérément... W.G. Sebald» prend en quelque sorte le parti de la négativité, de la détresse. L’art n'apaiserait pas la douleur, mais au contraire mettrait à nu les liens indissociables entre la création et la perte. À travers la pensée des philosophes Platon et Aristote, Bernier démontre comment il est difficile de se défaire de cette idée de l'écriture comme rédemptrice et réparatrice. C'est en compagnie de Sebald que l'auteur de cet article nous invite à faire dans la littérature l'expérience d'un «séjour au bord de l'anéantissement», "là où la perte nous tient comme notre danger et notre bien le plus propre».

Dans la partie Recherche de ce numéro, Marcelo Otero et Dahlia Namian s'appuient sur des données empiriques pour faire émerger l'ambivalence des patients quant à la prise de psychotropes. Les patients oscillant entre réticence et résignation quant à l'objet ambigu qu'est l'antidépresseur tantôt compris comme drogue, tantôt comme médicament. À la réticence s'ajoute la gamme de sentiments d'inconfort qui vont de la crainte de dépendance à la honte de se sentir dévalué par la prothèse humorale. La dépression a beau être une maladie dans l'air du temps, le diagnostic de dépressif n'en porte pas moins une dimension stigmatisante que les patients ressentent. La maladie mentale n'est pas une maladie comme les autres. Témoignages à l'appui, l'article procède à un fin dénombrement de l'échelle des résistances manifestées dans le discours des usagers devant la prescription de psychotropes.

Dans la section Points de vue, le docteur Jean-François Saucier dans un texte intitulé: "Augmentation significative de l'usage des antidépresseurs. Stratégies de contrôle, système médical dysfonctionnel ou besoin réel? » traite de la situation singulière que constitue l'augmentation du nombre des dépressions majeures en Amérique du Nord. Il rappelle dans un premier temps que: "l'Organisation mondiale de la santé a dû proclamer, récemment, que la dépression est devenue la deuxième cause de dysfonctionnement de la santé, juste après les maladies cardiovasculaires et avant le cancer». Il établit ensuite la liste des facteurs susceptibles d'avoir provoqué l'épidémie dépressive: baisse dans la consommation de poissons et donc d'oméga 3, recrudescence de l'obésité et du diabète, de l'épuisement professionnel, démembrement de la structure du monde du travail avec en corollaire l'effacement des réseaux de soutien.

Dans un second temps, pour penser la réalité épidémiologique de la crise, il analyse les causes structurelles en discutant l'hypothèse des erreurs de diagnostic. Il attribue la prolifération du diagnostic à un changement de mentalité quant aux rapports à la souffrance et à la difficulté dans le système médical actuel d'assumer les lenteurs d'une consultation psychiatrique. L'omnipraticien refuserait d'exposer plus longtemps son patient à sa détresse psychique et prescrirait l'antidépresseur. L'auteur suggère un certain nombre de pistes de réflexion pour atténuer cette anomalie épidémique en indiquant à chaque fois les limitations pratiques à ces suggestions constructives.

Les peurs du déprimé et les aléas des traitements qui lui sont proposés retiennent l'attention de Marc-Alain Wolf et de Yaël Wolf. Leur texte nous rappelle que l'antidépresseur reste un agent thérapeutique controversé, dont l'utilisation croissante est liée non seulement aux diagnostics posés par des cliniciens, mais aussi aux pressions qui s'exercent sur le marché des produits pharmaceutiques. Pour le patient déprimé, il n'est pas toujours facile de faire un choix éclairé et on peut comprendre qu'il se questionne sur l'utilité ou la nocivité d'un traitement. Les auteurs signalent que malgré les efforts visant à mieux contrôler et évaluer les traitements offerts, il faut tenir compte de ce qu'ils appellent «les pathologies de l'offre et de la demande». Parmi ces pathologies, ils mentionnent: "la prolifération anarchique de l'offre en psychothérapie, la crédulité sans limite de certains clients, le pouvoir excessif de l'industrie et du facteur économique dans la recherche et la clinique pharmacologiques, la confusion croissante entre le statut de patient et celui de consommateur».

Le texte de Nicolas Lévesque «La violence de l'objectivité» s'inscrit dans l'horizon de la pratique de psychologue de l'auteur. Il est marqué par l'attitude de retenue ironique qu'adopte le thérapeute à l'endroit des questions qui lui sont adressées par les patients sur l'efficacité des molécules. Le praticien y répond sous le mode humoristique par l'engouement pour Marineland, assimilant la médication au dressage ou au développement de réflexes conditionnés. Dans ce contexte, ce n'est ni l'innocuité ni l'efficacité présumée de la molécule qui intéresse le praticien. C'est le rapport de l'analysant au geste autoritaire de se voir prescrire qui, sous un autre registre, fait écho à sa propre histoire. Il en va toujours d'une «ordonnance à remplir» dans le petit papier énigmatique qu'apporte le patient sous prescription au pharmacien. Lévesque décrit ensuite à l'aide de récits de patients les lieux où le médicament remplace l'écoute, opère une mise au silence du sens des histoires personnelles. L'auteur refuse le temps «comprimé» celui-là même qui empêche l'intervention patiente et délicate dans le dénouement d'une histoire ou qui, plus simplement, affecte la capacité même de réfléchir aux conséquences de la surmédication des comportements. Nous irions d'une surdité involontaire à des stratégies d'évitement institutionnelles dans l'autojustification des rapports de pouvoir en place. Nicolas Lévesque perçoit l'époque des antidépresseurs non pas comme une évolution par rapport à des traitements encore plus frustres - comme si les armes atomiques constituaient un progrès de l'humanité par rapport à l'usage des baïonnettes - mais comme un dérèglement de la raison. L'article en appelle à une révolution qui soit tout incluse dans le cadre nouveau qu'offrirait une pensée de l'hospitalité, autrement dit, il demande de fixer des limites au déraisonnable au sein de la raison elle-même, de subvertir la raison calculatrice depuis le hors-prix de l'écoute. 